This item was submitted to Loughborough's Research Repository by the author.

Items in Figshare are protected by copyright, with all rights reserved, unless otherwise indicated.

\title{
Informational constraints, option generation, and anticipation
}

PLEASE CITE THE PUBLISHED VERSION

https://doi.org/10.1016/j.psychsport.2018.11.012

PUBLISHER

(c) Elsevier

VERSION

AM (Accepted Manuscript)

PUBLISHER STATEMENT

This paper was accepted for publication in the journal Psychology of Sport and Exercise and the definitive published version is available at https://doi.org/10.1016/j.psychsport.2018.11.012.

\section{LICENCE}

CC BY-NC-ND 4.0

\section{REPOSITORY RECORD}

Murphy, Colm P., Robin Jackson, and A. Mark Williams. 2018. "Informational Constraints, Option Generation, and Anticipation". Loughborough University. https://hdl.handle.net/2134/36183. 
9

10

Author Note

18 None of the authors have any links to companies or manufacturers who would benefit from this work. There are no conflicts of interest present. 
Objectives: To determine the option generation strategies used by expert and novice tennis players when anticipating an opponent's intentions using information that would normally be picked up sequentially prior to the opponent striking the ball.

Design and method: Altogether, 12 expert and 14 novice tennis players completed an option generation task when presented with rallies from real matches under two display conditions, namely video film and animations. Rallies were presented in such a way that participants either had access to contextual information and postural cues (video) or solely contextual information (animation; e.g., player positioning, shot sequencing).

Results: The experts were more accurate than novices in both display conditions. Participants generated less options in the video compared with the animated condition. More often than not, participants chose the first option they generated. The expert participants generated more task-relevant and fewer task-irrelevant options than novices, with this effect being stronger in the animated than the video condition. The number of options generated was negatively related to performance in the video condition only.

Conclusions: In dynamic, time-constrained tasks, performers adapt their option generation strategy depending on the information available. In keeping with Long Term Working Memory theory (Ericsson \& Kintsch, 1995), when constrained to anticipate based on contextual information alone, effective anticipation is underpinned by being able to access the likely outcome and potential relevant alternatives. Moreover, when pertinent postural cues become available, option generation strategies consistent with the Take The First heuristic model may be optimal (Johnson \& Raab, 2003). Implications for performance and training are discussed.

Keywords: perceptual-cognitive expertise; contextual information; tennis. 


\title{
Informational Constraints, Option Generation, and Anticipation
}

\author{
Colm P. Murphy ${ }^{1,2}$, Robin C. Jackson ${ }^{3}$, and A. Mark Williams ${ }^{4}$
}

${ }^{1}$ Department of Life Sciences, Brunel University London, Uxbridge, UB8 3PH, UK.

${ }^{2}$ Expert Performance and Skill Acquisition Research Group, Faculty of Sport, Health and Applied Science, St Mary’s University, Twickenham, London, TW1 4SX, UK.

${ }^{3}$ School of Sport, Health and Exercise Sciences, Loughborough University, Leicestershire, LE11 3TU, UK.

${ }^{4}$ Department of Health, Kinesiology and Recreation, University of Utah, Salt Lake City, UT 81442, USA.

Corresponding Author: Colm P. Murphy

Expert Performance and Skill Acquisition Research Group, Faculty of Sport, Health and Applied Science, St Mary’s University, Twickenham, London, TW1 4SX, UK.

Telephone: +447531696734

E-mail: colm.murphy@stmarys.ac.uk 
1

2

3

4

5

6

7

8

Informational Constraints, Option Generation, and Anticipation

9

10

11

12

13

14

15

16

17

Author Note

18 None of the authors have any links to companies or manufacturers who would benefit from

19 this work. There are no conflicts of interest present. 
1
2 Objectives: To determine the option generation strategies used by expert and novice tennis

3 players when anticipating an opponent's intentions using information that would normally be

4 picked up sequentially prior to the opponent striking the ball.

5 Design and method: Altogether, 12 expert and 14 novice tennis players completed an option

6 generation task when presented with rallies from real matches under two display conditions,

7 namely video film and animations. Rallies were presented in such a way that participants

8 either had access to contextual information and postural cues (video) or solely contextual

9 information (animation; e.g., player positioning, shot sequencing).

10 Results: The experts were more accurate than novices in both display conditions. Participants

11 generated less options in the video compared with the animated condition. More often than not, participants chose the first option they generated. The expert participants generated more task-relevant and fewer task-irrelevant options than novices, with this effect being stronger in the animated than the video condition. The number of options generated was negatively related to performance in the video condition only.

Conclusions: In dynamic, time-constrained tasks, performers adapt their option generation strategy depending on the information available. In keeping with Long Term Working Memory theory (Ericsson \& Kintsch, 1995), when constrained to anticipate based on contextual information alone, effective anticipation is underpinned by being able to access the likely outcome and potential relevant alternatives. Moreover, when pertinent postural

21 cues become available, option generation strategies consistent with the Take The First heuristic model may be optimal (Johnson \& Raab, 2003). Implications for performance and

23 training are discussed.

24 Keywords: perceptual-cognitive expertise; contextual information; tennis. 
2

In dynamic, fast ball sports such as tennis, the information available to performers

3 when trying to anticipate changes from moment to moment (Triolet, Benguigui, Le Runigo,

4 \& Williams, 2013). Anticipation may be largely based on contextual information such as shot

5 sequencing and player positioning (e.g., Abernethy, Gill, Parks, \& Packer, 2001; Loffing \&

6 Hagemann, 2014; Murphy, Jackson, \& Williams, 2018) or on a combination of contextual

7 information and postural cues such as the hip or shoulder movements of an opponent (e.g.,

8 Causer, Smeeton, \& Williams, 2017; Jackson \& Mogan, 2007; Ward, Williams, \& Bennett,

9 2002). The relative extent to which these two sources of information facilitate anticipation is

10 thought to vary as the critical event approaches (i.e., ball-racket contact by an opponent), with

11 contextual information being available prior to and during an opponent's movement pattern

12 while pertinent postural cues arise later in the action as the opponent shapes up to play the 13 decisive stroke (Buckolz, Prapavesis, \& Fairs, 1988; Farrow \& Abernethy, 2015; Müller \& 14 Abernethy, 2012). However, while our knowledge of how experts use postural cues to anticipate is well developed, our understanding of how contextual information is used to facilitate the process remains limited (Cañal-Bruland \& Mann, 2015).

In fast ball sports like tennis the time taken to process information and initiate a response often exceeds the time available to the player prior to ball arrival (Williams, Davids, \& Williams, 1999). For example, serve speeds of over $200 \mathrm{~km} \cdot \mathrm{h}^{-1}$ have been recorded (Mecheri, Rioult, Mantel, Kauffmann, \& Benguigui, 2016). It can therefore take less than 500 ms for the ball to travel from one player to the other (Abernethy \& Wollstein, 1989). Triolet et al. (2013) used video-based analyses to show that professional tennis players adapt their behaviour to deal with this time constraint by using different sources of information to anticipate. The researchers inferred the frequency of anticipation behaviours based on the timing of players' lateral movements relative to the opponent striking the ball. In favourable 
1 or neutral conditions (e.g., when the opponent was out of position or when rallying from

2 behind the baseline), responses appeared to be largely based on ball flight information as

3 players could afford the time to wait for this information to become available. However, in

4 unfavourable conditions (e.g., when the opponent was attacking from inside the court area)

5 players frequently began to respond well in advance of the opponent striking the ball,

6 implying that they were anticipating based either on contextual information (associated with

7 earlier occurring movements) or a combination of contextual and postural cues (associated

8 with later occurring movements). Moreover, the accuracy of the early-occurring anticipation

9 behaviours was much higher than chance, suggesting that contextual information may be used to guide anticipation. The authors concluded that expert performers use different types of information when trying to anticipate depending on the constraints present at that time.

To determine how athletes use postural information to anticipate, expert and novice

13 performers have been asked to make judgments based on footage of an opponent carrying out

14 an action in which the visual display was manipulated/occluded (e.g., Müller, Abernethy, Eid, Ward, \& Janelle, 2007). These cues appear to become available a few hundred milliseconds prior to the critical event. While the benefit of being able to effectively utilise postural cues is obvious, performers may not be afforded the time to wait for pertinent postural cues to become available under particularly extreme time constraints and therefore they could be constrained to anticipate based on contextual information alone (Triolet et al., 2013). 
1 Kamp, \& Ruiz, 2013), and shot sequencing (Murphy et al., 2018) to anticipate more

2 effectively than less-skilled counterparts. However, with few exceptions (e.g., Gredin,

3 Bishop, Broadbent, Tucker, \& Williams, 2018; McRobert, Ward, Eccles, \& Williams, 2011;

4 Milazzo, Farrow, Ruffault, \& Fournier, 2015), researchers have examined whether rather than

5 how contextual information is used to anticipate. It is thought that expert performers initially

6 use contextual information to generate expectations of what is about to happen based on

7 domain-specific knowledge, with these expectations being adjusted as more reliable

8 information (i.e., postural cues) becomes available (e.g., Gredin et al., 2018; Loffing \&

9 Hagemann, 2014). Since the information available to performers changes over time as the critical event (e.g., ball-racket contact) approaches, research is required to examine the mechanisms underpinning expert anticipation when constrained to anticipate based on contextual information alone compared with when pertinent postural cues are available.

A common approach has been to present participants with potential alternatives from

14 which to choose what the opponent is most likely to do next (e.g., Bourne, Bennett, Smeeton,

15 Hayes, \& Williams, 2013; Loffing, Sölter, Hagemann, \& Strauss, 2015; Roca, Ford,
McRobert, \& Williams, 2011). However, in real-world situations, performers must search for information, assess the situation and generate potential alternative courses of action. The option generation paradigm was initially employed by Johnson and Raab (2003) to examine how performers generate and choose options when making decisions about what course of action to take. This paradigm has subsequently been used to investigate the option generation strategies underpinning both anticipation (e.g., Belling, Suss, \& Ward, 2015a; Ward, Ericsson, \& Williams, 2013) and decision making (e.g., Raab \& Laborde, 2011; Ward, Suss, Eccles, Williams, \& Harris, 2011). In the natural ecology both judgements involve the generation of options. The few researchers who have investigated both anticipation and 
1 decision making in the same study have demonstrated similar trends in the option generation

2 strategies underpinning each judgement (e.g., Belling et al., 2015a; Ward et al., 2011).

4 particularly evident in the need to understand how expert performers generate and choose

5 between potential alternatives in situations involving extreme time constraints. In such

6 instances, the outcome of the upcoming event is often highly uncertain, and performers are

7 likely to initially generate options based on their expectations of what might happen given the

8 contextual information available. When constrained to form expectations based on contextual

9 information alone, there is likely to be more than one plausible outcome, whereas at the moment of the critical event occurring (i.e., ball-racket contact) the number of potential outcomes is likely to have decreased, given the increasing availability of postural cues.

In their seminal research on option generation, Johnson and Raab (2003) proposed the 'Take The First' (TTF) as an heuristic that is used to generate and choose between options in divergent thinking situations. The development and application of TTF was motivated by research advocating the use of simple heuristics such as Fast and Frugal (Gigerenzer \& Todd, 1999). Advocates of such heuristics suggest that deliberation is not a necessary part of the decision making process. Simple rules of thumb suffice for making appropriate decisions. Limited deliberation would certainly appear beneficial in fast-ball sports, where performance frequently occurs under extreme time constraints (Raab, 2012). Specifically, the TTF heuristic model (Johnson \& Raab, 2003) proposes the use of associative memory networks and fast and frugal heuristics to explain how expert performers make decisions in domainspecific situations. It is suggested that learned associations between situations and options form, with the association strengthening if the option is repeatedly chosen. The model further assumes that options are generated sequentially and that those options with the strongest association are generated first. The TTF heuristic therefore advocates a "less-is-more" option 
1 generation strategy. It predicts that a negative relationship will exist between the number of

2 options generated and the quality of the final option chosen, and that generating fewer

3 options will result in a higher quality final choice than if more options are generated. Johnson and Raab (2003) presented this model based on a divergent task completed

5 by intermediate level handball players. After viewing videos from a handball match, which

6 were frozen after 10 seconds, participants were instructed to quickly identify the first option

7 that intuitively came to mind, then as many options as they could conceive, and finally to

8 choose the option they considered to be the best for the specific situation. A negative

9 relationship was reported between the total number of options generated and the quality of

10 their final choice (based on the judgment of an expert panel). Moreover, approximately $60 \%$

11 of the time, participants chose the first option they generated. Finally, although not a primary

12 aim of their study, the authors observed a significant positive correlation between experience

13 and the quality of the final option chosen. Raab and colleagues (e.g., Laborde \& Raab, 2013;

14 Raab \& Johnson, 2007; Raab and Laborde, 2011) have subsequently reported that experts generate higher quality options as their first option than less expert performers.

Ward et al. (2013) proposed that Long Term Working Memory theory (LTWM;

Ericsson \& Kintsch, 1995) could support option generation in complex tasks. According to this theory, due to extensive experience and practice within a domain, expert performers acquire elaborate domain-specific memory representations. Information is proposed to be

21 both the likely outcome of upcoming events as well as potential relevant alternatives. LTWM theory therefore proposes fundamentally different mechanisms for effective decision making to those of the TTF heuristic. Whereas TTF would suggest that the encoding of more options 24 would result in a decrease in decision quality, LTWM theory proposes that, if multiple taskrelevant options exist, generation of these options will result in higher quality decisions. The 
1 adaptability and flexibility associated with the generation of more than one task-relevant 2 option could be particularly important in dynamic, complex tasks (Hoffman et al., 2014). Ward et al. (2013) required skilled and less-skilled soccer players to anticipate the

4 opponent's intentions after viewing video footage of sequences from matches. Participants

5 generated all options they thought the opponent might take as well as indicating what they

6 thought the opponent would actually do. To enable the researchers to test the predictions of

7 LTWM theory (Ericsson \& Kintsch, 1995), a panel of expert coaches classified options as

8 task-relevant or task-irrelevant. Task-relevant options were deemed to be of specific

9 relevance, given the goal of the task. For example, when in an attacking situation in soccer, a constraint affects option generation when anticipating the opponent's intentions and when making a decision about the course of action to take. In the time-constrained condition, participants had 10 seconds to complete the task, whereas in the other condition, no time constraint was enforced. The skilled participants generated more task-relevant and fewer task-irrelevant options than less-skilled participants in both the anticipation and the decision making task. In the decision making task, fewer task-irrelevant options were generated in the 
1 time-constrained compared with the non-time-constrained condition; however, no differences

2 between conditions were observed for the anticipation task. This selective reduction of task-

3 irrelevant options in the decision making task was reported to be consistent with the TTF

4 heuristic model. However, the significant negative correlation between the number of options

5 generated and performance, which would be predicted by the TTF heuristic model, was not

6 observed in either condition. Yet, significant positive and negative correlations between task-

7 relevant and task-irrelevant options and performance (selection of the criterion best option as

8 judged by an expert panel) were observed. Belling et al. (2015a) suggested that participants

9 used a strategy that was more consistent with LTWM theory than the TTF heuristic model,

both when anticipating the opponent's intentions and when making a decision about what

course of action to take. Nevertheless, the reduction in task-irrelevant options generated under time constraint in the decision making task suggests that the option generation strategy employed is at least somewhat dependent on the task constraints.

Several researchers have now examined the effect of task constraints on anticipation (e.g., Cocks, Jackson, Bishop, \& Williams, 2015; North, Hope, \& Williams, 2016; Roca, Ford, McRobert, \& Williams, 2013). Murphy et al. (2016) examined how anticipation accuracy in skilled and less-skilled tennis players differed depending on the information presented. Participants viewed the same rallies from real tennis matches in either animated format, which omitted the bodies and rackets of the players such that participants were constrained to anticipate based on contextual information alone (as would be necessary in a real match when placed under particularly extreme time constraints and the player cannot afford to wait for pertinent postural cues to become available), or video format, in which both contextual information and pertinent postural cues were available. While participants were able to accurately anticipate the opponent's intentions based on contextual information alone, 
1 they were more accurate when postural cues were also available. Moreover, skilled

2 participants were more accurate regardless of the information available.

The cognitive mechanisms underpinning expert anticipation may differ depending on

4 the information available. Müller and Abernethy (2012) proposed a model of anticipation

5 based on the temporal pick-up and use of information in striking sports. The proposal is that

6 contextual information available prior to the opponent commencing the final stroke acts to

7 prime the performer's response when the pertinent postural cues subsequently become

8 available. As the availability of postural cues increases as ball contact approaches, the

9 number of action possibilities decreases. In this paper, we compare the option generation contextual information alone followed by both contextual and postural information).

\section{Anticipation Accuracy}

effectively pick up and utilise pertinent postural cues from the opponent.

\section{Option Generation: Number and Type of Options Generated}

We predicted, based on previous published reports (Johnson \& Raab, 2003; Ward et al., 2013), that participants would generate relatively few options per trial. Moreover, we 
1 contextual and postural information than when presented only with contextual information

2 due to the availability of postural cues and resultant reduction of action possibilities (Müller

$3 \&$ Abernethy, 2012). We further hypothesized that this reduction would be more pronounced

4 in expert than novice participants due to their greater ability to pick up and utilize pertinent

5 postural cues (Mann et al., 2007). We test Müller and Abernethy's (2012) assertion that

6 expert performers reduce the action possibilities of the opponent over time as prioritisation of

7 information sources shifts from the use of contextual to postural information, whereas novice

8 performers lack the ability to prioritise information sources as effectively.

We hypothesized, based on the findings of Ward et al. (2013) and Belling et al. (2015a), and in support of LTWM theory (Ericsson \& Kintsch, 1995), that expert participants

would generate more task-relevant and fewer task-irrelevant options than novices. We expected this effect to be more pronounced when anticipating based solely on contextual

13 information as the complexity of the task would be greater, potentially making adaptability

14 and flexibility more important (Hoffman et al., 2014).

\section{Option Generation: Frequency of Taking the First Option}

If the predictions of TTF (Johnson \& Raab, 2003) were to hold true, we expected

17 participants to more often than not choose the first option generated.

\section{Relationship between Option Generation and Anticipation Accuracy}

We hypothesized, based on previous research on option generation and anticipation

(Belling et al., 2015a; Ward et al., 2013), that a higher number of task-relevant options and lower number of task-irrelevant options would be related to more accurate anticipation (Belling et al., 2015a; Ward et al., 2013). 


\section{Methods}

\section{Participants}

Altogether, 12 expert $\left(M_{\text {age }}=24.33, S D=4.48\right)$ and 14 novice $\left(M_{\text {age }}=24.45, S D=\right.$

4 5.41) male tennis players participated. The expert participants had played tennis for a mean of

$5 \quad 18.25$ years $(S D=6.02)$, whereas novices play recreational tennis irregularly and had never

6 played competitively. We calculated sample size using G*Power 3.1 (Faul, Erdfelder, Lang,

$7 \quad \&$ Buchner, 2007). Based on previous findings using the same form of test stimuli and similar

8 expertise levels of participants (Murphy et al., 2016), 15 participants were shown to be

9 sufficient to detect a large effect size $\left(f=0.80, \eta_{p}^{2}=0.39\right)$ across the two independent

10 variables, with a power of 0.80 and an alpha level of .05. The experts held British Tennis

11 ratings of between 1.1 and 4.1, whereas the novices did not hold a rating. The rating system is

12 based on competition results with the highest rating possible being 1.1 and the lowest rating

13 being 10.2. One participant in each group was left-hand dominant. All participants had

14 normal or corrected to normal vision. Written informed consent was received from each of

15 the participants and ethical approval was obtained from the lead university's ethics board.

\section{Test Stimuli}

Test stimuli were created from points played in professional men's tennis matches at the AEGON Championships (2013). The video condition was created from video footage recorded at $30 \mathrm{~Hz}$ using a wide angle lens camera (Contour Roam, Contour Inc., Seattle, USA) from a height of $1.9 \mathrm{~m}$ above the ground and $6.4 \mathrm{~m}$ behind the court. Player movement and ball trajectory data (Hawk-Eye Innovations Ltd., Basingstoke, UK), taken from the same rallies used in the video condition, were input into a rendering engine (Julien Pansiot, London, UK) to generate footage for the animated condition (see Murphy et al., 2016). The animated footage omitted the players' bodies and rackets from the visual display such that 
1 participants would be constrained to anticipate based on contextual information alone (such

2 as the relative movements of the players, their positioning, and shot sequencing). This display

3 condition was designed to simulate instances in real tennis matches where players cannot

4 afford to wait for pertinent postural cues to become available (e.g., under particularly extreme

5 time constraints) and therefore must anticipate based on contextual information alone. In

6 these animations, the two players were presented as a blue and a red cylinder, their rackets

7 were not visible, and the ball was presented as a yellow dot. Video footage on the other hand

8 displayed both postural and contextual information as the footage was not manipulated in any

9 way (see Figure 1). In both display conditions, trials were edited to occlude at the opponent's ball-racket contact using Pinnacle Studio 15 editing software (Pinnacle, Ottawa, Canada).

\section{$<<$ Insert Figure 1 near here $>>$}

Situations in which the player on the near side of the court was placed under extreme time constraints were selected as test stimuli (as per Murphy et al., 2016). Tennis players have been shown to anticipate more often when the balance of power is unfavourable and the time-constraints are more extreme, such as when the opponent is attacking from the inside of the court (Triolet et al., 2013). The final occluded shots of trials were therefore made up of attacking drives, passing shots, drop-shots, and volleys. Each of these shots would imply that the player receiving the shot was performing under extreme time constraints, the balance of power in the rally was unfavourable, or both. The experimental trials were made up of 14 rallies from nine different matches in various rounds of the tournament, featuring 14 different players, all of whom were right-handed. Trials began three seconds prior to the serve being hit and contained between two and seven shots prior to occlusion at the opponent's ballracket contact. From the moment the serve was struck until the moment of occlusion, trials lasted an average of $4.06 \mathrm{~s}(S D=2.69)$. The 14 trials used in the study had been used in a previous study (Murphy et al., 2016). 
Prior to data collection, two expert coaches with an average of 15 years of experience

2 in tennis, at national and international level, watched all of the animated and video sequences.

3 The coaches identified all areas of the court the receiving player should be concerned about

4 the ball being hit to. On an A4 piece of paper, with a scaled schematic of a tennis court on it,

5 the coaches were asked to identify these areas based on the information that they had

6 available to them from the display up until the point of occlusion. These areas of the court

7 would therefore represent task-relevant options for the anticipation task (see Belling et al.,

8 2015a). The schematic of the tennis court had a grid of 16 (width) by 23 (length) squares on it

9 to allow coaches to easily designate these areas of the court.

To ensure that all possible task-relevant options were identified, the coaches could watch the trials as many times as they wished. Coaches completed this process at the same time, but did not see each other's responses until both had finished. Immediately after identifying the areas of the court they considered relevant, they had a discussion about the areas of the court they identified as task-relevant options and their reasons for doing so. The level of agreement between the two coaches in video and animated conditions was $81.2 \%$ and $87.4 \%$ respectively. On a separate schematic, areas of the court the coaches agreed upon were then outlined. Any other areas of the court that were not agreed upon were disregarded. This resulted in 34 and 52 relevant areas of the court being identified in the video and animated conditions respectively. The lesser number of task-relevant options identified in the video condition was expected based on Müller and Abernethy's (2012) model of anticipation, suggesting that the number of action possibilities are reduced as pertinent postural cues become available. It was considered important that the expert coaches identify task-relevant options in both rather than just one condition, as this would permit evaluation of the options generated by participants, relative to the information available in each condition. The options generated by participants that were located inside an area of the court identified by the 
1

coaches were deemed task-relevant options, whereas those located outside these areas were

2 deemed task-irrelevant.

3 Materials, Apparatus, and Set-Up

4

5 Participants sat approximately $40 \mathrm{~cm}$ from the screen yielding a viewing angle of the

6 opposing player of around $2^{\circ}-3^{\circ}$ (for similar viewing angles, see Cocks et al., 2015; Murphy

$7 \quad$ et al., 2016).

\section{Procedure}

Participants viewed eight familiarization (four video and four animated trials) and 28 experimental trials (14 video and the 14 corresponding animated trials), with none of the familiarization trials being used as experimental trials. In the experimental trials, the same 14 open-play rallies with the same outcome were presented in video and animated format such that comparisons could reliably be made between response accuracy and option generation strategies across the two conditions (as per Murphy et al., 2016). To control for order effects, six sets of the 28 trials (14 trials in each condition) were created for use as test stimuli. The same trials were used in each set, but the trials were presented in a different, randomly generated order in each set. Each set of trials was viewed by four participants (two experts and two novices), except for two of the sets which were viewed by three novice and two expert participants. Each set consisted of four blocks of seven trials with participants having a one-minute break in between blocks to prevent boredom. In the experimental trials, the first bounce of the ball after the shot on which the trial was occluded was deep (past the service line) on ten trials and short (before the service line) on 4 trials. In half of these trials, the ball was directed to the left side of the court and in the other half to the right side of the court (see supplementary material for a schematic overview of ball bounce locations). 
Participants received the same instructions as to how to complete the task. They were

3 the display would be occluded (i.e., the screen would go black) at the moment of the

4 opponent's ball-racket contact. In contrast to the expert coaches, participants were only

5 allowed to view trials once prior to responding. They were told that at the moment of

6 occlusion, on a scaled down image of a tennis court on a piece of A4 paper, they should

7 indicate the expected ball bounce location of each of the shots they thought the opponent

8 might hit (by drawing an ' $\mathrm{X}$ ' on the court). Moreover, they were told that the objective of the

9 task was not to provide an exhaustive list of every possible option they could think of but to indicate only the options they thought of at the moment the clip was occluded. For each option generated, participants were asked to rate the likelihood, on a scale of 0 to 10 , with 0 being not at all likely and 10 being certain, that the opponent would hit the shot where the ' $\mathrm{X}$ ' was placed. The ' $\mathrm{X}$ ' that received the highest likelihood score was considered their anticipated option (see Belling et al., 2015a). If their anticipated option (the option that received the highest likelihood score) landed in the same quadrant of the court as the ball actually bounced it was considered correct (see Murphy et al., 2016). The primary researcher noted the order in which participants generated options. A percentage of correct judgments was calculated for anticipation judgments (correctly anticipating the quadrant of the court in which the ball would bounce). Spearman-Brown coefficients of reliability for anticipation accuracy were 0.74 in the animated condition and 0.83 in the video condition.

\section{Data Analysis}

First, to determine whether there were differences in anticipation accuracy across display conditions and skill groups, we conducted a 2 (Display [video, animated]) $\times 2$ (Group [expert, novice]) ANOVA. Second, to examine whether the number and type of generated options differed between groups and display conditions, we conducted a 2 (Display) $\times 2$ 
1 (Group) $\times 2$ (Option Type [task-relevant, task-irrelevant]) ANOVA. To determine whether

3 the first option generated was ultimately chosen in each display condition. Finally, to

4 examine relationships between numbers and types of options generated and anticipation

5 accuracy, we conducted multiple regressions and follow-up Pearson's correlations. In the

6 case of significant interactions, pairwise comparisons were conducted. The alpha level was

7 set at .05. Partial eta squared $\left(\eta_{p}^{2}\right)$ and Cohen's $d$ were used to report effect size. Any

8 violations of sphericity were corrected with Greenhouse-Geisser procedures and in the case

9 of multiple $t$-tests, Sequential Bonferroni was applied. All analyses were conducted using

IBM SPSS Statistics (version 20).

\section{Results}

\section{Anticipation Accuracy}

The results of the 2 (Display) $\times 2$ (Group) ANOVA are presented in Table 1 . The mean anticipation accuracy and standard error scores for expert and novice participants are presented in Figure 2. ANOVA revealed a significant main effect of Display. Anticipation accuracy was higher in the video $(M=52.47, S D=20.10$, Range $=14.29-92.86)$ than the animated condition $(M=43.68, S D=11.49$, Range $=21.43-57.14)$. A main effect of Group was observed. Expert participants were more accurate overall than novices (expert: $M=$ $59.23, S D=8.40$, Range $=46.43-75.00$; novice: $M=38.52, S D=12.31$, Range $=17.86-$ 60.71). A significant Display $\times$ Group interaction was observed. Paired samples $t$-tests revealed that the accuracy of the expert participants increased significantly from the animated $(M=50.00, S D=7.46$, Range $=35.71-57.14)$ to the video condition $(M=68.45, S D=11.58$, Range $=57.14-92.86, p<.01, d=1.89$ ), whereas no difference was evident between the two 
1 conditions for the novice participants (animated: $M=38.27, S D=11.76$, Range $=21.43$ -

57.14; video: $M=38.78, S D=15.01$, Range $=14.29-64.29, p=.87, d=0.04)$.

$<<$ Insert Table 1 near here $>>$

$<<$ Insert Figure 2 near here $>>$

\section{Option Generation: Number and Type of Options Generated}

The results of the 2 (Display) $\times 2$ (Group) $\times 2$ (Option Type) ANOVA are presented

7 in Table 1. Only two of the participants generated more than three options on any trials so to avoid skewing the data, these options were removed from the analysis. The mean numbers

9 (and standard error) of task-relevant and task-irrelevant options for expert and novice

relevant options identified by the expert coaches, and consequently the number of task-

relevant options that participants could generate, was fewer in the video (34) than the

animated condition (52), we checked whether this effect was still observed when the

frequency of task-relevant options relative to the total number of possible task-relevant

options was analysed. ANOVA revealed a significant main effect of Display, with

participants generating fewer task-relevant options in the video than the animated condition,

$21 \quad F(1,24)=8.39, \mathrm{p}<.01, \eta_{p}^{2}=.26$

A significant Group $\times$ Option Type interaction was observed. Follow-up $t$-tests 
1 Range $=0.64-1.43$, novice: $M=0.76, S D=0.23$, Range $=0.43-1.07, p<.01, d=1.73)$ and

$$
3=0.33 \text {, Range }=0.61-1.71, p<.01, d=1.73) \text { options than novice participants. }
$$

4 A significant Display $\times$ Option Type interaction was observed. Participants generated 5 more task-relevant options in the animated condition than in the video condition (animated: $6 \quad M=1.07, S D=0.39$, Range $=0.43-1.71$, video: $M=0.80, S D=0.23$, Range $=0.29-1.14, p<$ $7.01, d=0.84)$, but no difference in the number of task-irrelevant options generated was 8 observed between the two conditions (animated: $M=0.90, S D=0.40$, Range $=0.36-1.50$, 9 video: $M=0.95, S D=0.41$, Range $=0.36-2.00, p=.17, d=0.12$ ).

ANOVA revealed a significant Display $\times$ Group $\times$ Option Type interaction. To further investigate the source of this interaction, an ANOVA was run in each display condition. The Group $\times$ Option Type interaction was significant in both the video, $F(1,24)=18.83, p<.01$, $\eta_{p}^{2}=.44$, and the animated condition, $F(1,24)=37.24, p<.01, \eta_{p}^{2}=.61$. Independent $t$-tests

revealed that while experts generated more task-relevant and fewer task-irrelevant options in both conditions the differences between groups were smaller in the video condition (relevant: $p<.01, d=1.25$, irrelevant: $p<.01, d=1.30$ ) than in the animated condition (relevant: $p<$ $.01, d=1.75$, irrelevant: $p<.01, d=1.86$ ). Pairwise comparisons revealed that neither experts (animated: $M=0.60, S D=0.29$, Range $=0.36-1.36$, video: $M=0.71, S D=0.23$, Range $=0.36-1.14, p=.06, d=0.42$ ) nor novices (animated: $M=1.15, S D=0.30$, Range $=$ $0.64-1.50$, video: $M=1.15, S D=0.42$, Range $=0.57-2.00, p=.48, d=0.00)$ differed in the number of task-irrelevant options generated between the animated and the video condition. However, expert participants reduced the number of task-relevant options generated between the animated $(M=1.35, S D=0.26$, Range $=0.79-1.71)$ and video conditions $(M=0.93, S D=$ 0.19 , Range $=0.50-1.14, p<.01, d=1.84$ ) more than novice participants (animated: $M=$ 
$10.83, S D=0.33$, Range $=0.43-1.43$, video: $M=0.68, S D=0.21$, Range $=0.29-1.07, p=.04$, $2 d=0.54)$. No other significant main effects or interactions were observed.

\section{Option Generation: Frequency of Taking the First Option}

6 novice: $77.30 \%$ ). In the video condition, participants chose the first option generated on

$7 \quad 85.08 \%$ of trials (expert: $91.07 \%$; novice: $79.08 \%$ ), whereas in the animated condition they

8 did so on $78.83 \%$ (expert: $75.51 \%$; novice: $82.14 \%$ ) of trials.

\section{Relationship between Option Generation and Anticipation Accuracy}

The results of the correlational analyses are reported in Table 2. Additionally, the

relationships between option generation and anticipation accuracy are presented in Figures 4 and 5. A significant negative relationship was observed between the number of options generated and anticipation accuracy in the video condition, but not the animated condition.

14 Pearson's correlations within skill groups revealed no significant relationships between number of options generated and accuracy for either group in the video condition or the animated condition, with these negative relationships being stronger for the novices than the expert participants.

$<<$ Insert Table 2 near here $>>$

Multiple regression revealed that the number of task-relevant and task-irrelevant

21 options significantly predicted anticipation accuracy in the video condition $\left(R^{2}=.41, F(2,25)\right.$

$22=7.94, p<.01)$. Pearson's correlations revealed a moderate, significant positive relationship 
1 between the number of task-relevant options generated and accuracy and a strong, significant

2 negative relationship between the number of task-irrelevant options generated and accuracy.

3 The number of task-relevant and task-irrelevant options significantly predicted accuracy in

4 the animated condition $\left(R^{2}=.36, F(2,25)=6.33, p<.01\right)$. Pearson's correlations revealed a

5 moderate, significant positive relationship between the number of task-relevant options

6 generated and accuracy, and a strong, significant negative relationship between the number of

7 task-irrelevant options generated and accuracy.

8

$$
<<\text { Insert Figure } 5 \text { near here }>>
$$

\section{Discussion} pattern (Mann et al., 2007). The main effect of Display suggests that anticipation accuracy was higher overall in the video than the animated condition. However, the Display by Group

We compared the option generation strategies employed by expert and novice tennis players when completing a task in which they were required to anticipate the outcome of an opponent's shot based on contextual information alone (animations) or when both postural and contextual information (videos) was available. We expected, based on previous published reports, to observe differences in anticipation accuracy (Abernethy et al., 2001; Loffing \& Hagemann, 2014; Murphy et al., 2016) and option generation strategies (Belling et al., 2015a; Johnson \& Raab, 2003; Ward et al., 2013) between skill groups and display conditions.

As expected, based on the findings of Murphy et al. (2016), experts were more accurate than novices overall, with this difference in accuracy being more pronounced in the video condition. This finding was expected because experts have been consistently shown to be more effective than novices in utilising contextual information (Farrow \& Reid, 2012; Murphy et al., 2016) and in picking up pertinent postural cues from an opponent's movement interaction reveals that this effect was driven by the expert participants' large increase in 
1

4 considered the available contextual information to be more reliable. The latter explanation is 5 in line with recent findings that suggest expert performers can prioritise the use of the most 6 reliable information sources to optimise anticipation (Gredin et al., 2018; Loffing et al., 7 2014). Overall, these findings support previous published reports (e.g., Abernethy et al., 8 2001; Murphy et al., 2016) and suggest that contextual information can be used to anticipate 9 highly effectively, yet if expert performers are afforded the time to wait for pertinent postural cues to become available, doing so can result in more accurate anticipation. availability of pertinent postural cues in addition to contextual information would result in a

As hypothesized, based on Müller and Abernethy's (2012) suggestion that the reduction in action possibilities, participants generated fewer options when viewing rallies in the video than the animated condition. Participants appear to adapt their option generation strategy depending on the information they have available to them at a given moment in time. This finding supports research which reports that skilled athletes prioritise relevant information based on its reliability as it becomes available (Gredin et al., 2018; Loffing et al., 2014). Gredin et al. and Loffing et al. found that far in advance of the critical event, skilled athletes prioritise contextual information, with a shift towards the prioritisation of postural cues occurring on approach to the critical event. Our findings could represent how this behaviour is supported by option generation strategies. When tennis players are constrained to anticipate based on contextual information in advance of pertinent postural cues becoming available (Buckolz et al., 1988; Farrow \& Abernethy, 2015; Müller \& Abernethy, 2012), they appear to generate all likely options. As the movement pattern of the opponent develops, pertinent postural cues are picked up which results in some previously considered options 
1 being deemed highly unlikely and disregarded. Consequently, players have fewer options to

3 strategy is consistent with Müller and Abernethy's (2012) model of anticipation.

$4 \quad$ We had further predicted, based on Müller and Abernethy's (2012) model that the

5 reduction in the number of options generated from the animated to the video condition would

6 be more pronounced for expert than novice participants. In particular, novice participants

7 were not expected to be able to use the available postural cues to reduce the opponents'

8 action possibilities in the same way as experts. While this effect was in the expected

9 direction, the Display by Group interaction was not significant. While the following

10 explanation is purely speculative, potentially the third person viewing angle used in our task

11 made participants more reliant on contextual information (picked up from the relative

12 movements/positioning of the players and shot sequencing) than they normally would be in

13 competition, reducing the degree to which they prioritised pertinent postural cues in the video 14 condition (Roca et al., 2013).

Consistent with the findings of Ward et al. (2013) and Belling et al. (2015a), and in

16 line with our hypothesis, expert participants generated significantly more task-relevant and

17 fewer task-irrelevant options than novice participants. This finding provides support for the 18 notion that expert performers develop elaborate domain-specific memory representations 19 which allow them to encode and maintain access to task-relevant information (Ericsson \& 20 Kintsch, 1995). This is thought to facilitate high levels of flexibility and adaptability 21 (Hoffmann et al., 2014). We further hypothesized that this effect would be stronger in the animated than in the video condition because of the potential need to be more adaptable in complex and less predictable situations, such as when constrained to anticipate based on 24 contextual information in advance of pertinent postural cues becoming available (Hoffman et al., 2014). The associated effect size for this interaction was larger in the animated than in the 
1

2 performers sometimes have to do in competitive situations prior to pertinent postural cues

3 becoming available (Triolet et al., 2013), an option generation strategy consistent with

4 LTWM theory which allows access to both the perceived most likely outcome and potential

5 relevant alternatives may be optimal.

Participants reduced the number of task-relevant options generated, but not taskirrelevant options, from the animated to the video condition. These findings differ from those of Belling et al. (2015a) who observed that participants reduced the number of task-irrelevant options generated when making decisions under time constraint; however, recall that no such differences were observed in the anticipation task they conducted. Our findings suggest that the number and type of options performers generate differ depending on the informational constraints. While the findings of Belling et al. may have been expected because reducing the number of task-irrelevant options generated when under time constraint would result in a higher proportion of relevant options from which to base a quick and accurate decision, our findings indicate that the number of task-relevant options performers generate depends on the information available at that moment. The higher anticipation accuracy scores in the video when compared with the animated condition indicate that reducing the number of taskrelevant options generated when postural cues were available was an effective strategy.

In support of the TTF heuristic model (Johnson \& Raab, 2003), participants more often than not chose the first option generated. Moreover, the percentage of trials on which participants chose the first option was higher than in previous studies $(81.95 \%$ compared to approximately 60\%, e.g., Johnson \& Raab, 2003; Raab \& Johnson, 2007). However, it is important to consider that this difference may be due to the nature of the task. For example, in our task participants noted potential options using pen and paper, whereas in other studies a verbal response has been used. Nevertheless, the high percentage of occasions on which 
1 participants chose the first option generated provides support for the notion that more often

2 than not, athletes "go with their gut", choosing the first option they generate rather than

3 subsequently generated options (Johnson \& Raab, 2003). While the data are merely

4 descriptive in nature, the finding that experts choose the first option generated more often

5 than novices $(86.61 \%$ vs $77.30 \%)$ suggests that this strategy may be employed more

6 frequently with increasing expertise.

The findings presented thus far appear to provide a somewhat mixed picture as to the 8 option generation strategies that underpin expert anticipation. The correlational data provide 9 some explanation as to why this may be the case. If expert anticipation is underpinned by a 10 "less-is-more" option generation strategy, as proposed by TTF (Johnson \& Raab, 2003), we 11 would expect the number of options generated to be negatively related to anticipation 12 accuracy. The correlational data suggest that the use of such strategies may be dependent on 13 the information available when anticipating. In the video condition, consistent with the TTF 14 heuristic model (Johnson \& Raab, 2003), a negative relationship was observed between the number of options generated and anticipation accuracy, whereas this relationship was not cues which can be picked up from an opponent to anticipate (e.g., Causer et al., 2017; Huys et al., 2009; Jackson \& Mogan, 2007). Therefore, if one is proficient in picking and up and utilising these cues, generating the low number of options associated with these cues and not 22 further generating options based on contextual information is likely to yield success. generated and performance in the video condition appears to have been driven by the novice participants. From a theoretical perspective, given the expert participants' experience levels, 
1 the associations between the presented kinematic cues and the most likely outcome are likely

2 to be strong enough for those athletes not to generate many other options, whereas for

3 novices these associations may be weak or poorly formed, resulting in the generation of

4 other, ultimately less likely options. In the animated condition, on the other hand, the strength

5 of the associations between a presented situation and the most likely outcome are likely to be

6 weaker given the high levels of uncertainty in the absence of kinematic cues. As such, the

7 generation of more task-relevant options appears to allow experts the required flexibility in

8 their response. However, regardless of the information presented, in both the video and

9 animated condition, anticipation accuracy levels were positively and negatively related to the number of task-relevant and task-irrelevant options generated respectively. We suggest that

11 while a less-is more strategy (e.g., TTF) may be effective in some situations (in this instance,

12 in situations of greater certainty), it nevertheless appears beneficial for the performer to generate and maintain access to potential relevant alternatives regardless of the information available (as prescribed by LTWM theory). This strategy may be particularly important in situations in which the outcome is not entirely congruent with the postural cues presented in

16 the lead up to the critical event, for example, due to varying levels of disguise or deception 17 (Jackson, Warren, \& Abernethy, 2006; Sebanz \& Shiffrar, 2009).

Our research findings provide support for the notion that perceptual-cognitive training 19 in complex, dynamic tasks should be context-specific (Hoffman et al., 2014). While Belling et al. (2015a) suggested that the option generation strategies employed when making decisions are affected by time constraints, we have demonstrated that the option generation

22 strategy employed when anticipating is dependent on the information available. We

23 recommend that training programmes be designed to reflect these differences. For example,

24 performers should be trained to pick up and utilize both contextual and postural information 25 to inform their judgments. This is particularly important, given that depending on the time 
1 constraints, they will often have to base their judgments on one or both of these types of

2 information. For situations in which performers may be constrained to anticipate based on

3 contextual information alone (e.g., when performing under particularly extreme time

4 constraints), it appears that training should prioritise the generation of all available task-

5 relevant options. However, training for situations in which pertinent postural cues as well as

6 contextual information are available appears less straight forward. On the one hand, it

7 appears that the generation of all available task-relevant options is a sensible strategy, yet on

In summary, in this paper we extend knowledge of the mechanisms underpinning expert anticipation. Specifically, we shed light on how expert performers generate and select options when different information sources are available. We demonstrated that the option generation strategies employed by expert performers are dependent on the information they are constrained to use to anticipate. In keeping with LTWM theory (Ericsson \& Kintsch, 1995), when constrained to anticipate based on contextual information prior to pertinent postural cues arising, generation of both the most likely option and alternative task-relevant options appears to underpin expert anticipation. However, as pertinent postural cues become available on approach to the critical event, a reduction in the number of options generated and considered facilitates more accurate anticipation. In such instances, when performers can afford to wait for pertinent postural cues to become available, generation of less options, and potentially a switch towards a strategy consistent with more intuitive models such as the TTF heuristic (Johnson \& Raab, 2003) may be optimal. Our findings highlight the dynamic and interactive nature of perceptual-cognitive expertise. 


\section{References}

2 Abernethy, B., Gill, D., Parks, S., \& Packer, S. (2001). Expertise and the perception of 3 kinematic and situational probability information. Perception, 30, 233-252.

4 Abernethy, B., \& Wollstein, J. (1989). Improving anticipation in racquet sports. Sports Coach, 12, 15-18.

6 Belling, P. K., Suss, J., \& Ward, P. (2015a). Advancing theory and application of cognitive research in sport: Using representative tasks to explain and predict skilled anticipation, decision-making, and option-generation behavior. Psychology of Sport and Exercise, 16, 45-59.

Belling, P. K., Suss, J., \& Ward, P. (2015b). The effect of time constraint on anticipation, decision making, and option generation in complex and dynamic environments. Cognition, Technology \& Work, 17, 355-366.

Bourne, M., Bennett, S., Hayes, S., Smeeton, N., \& Williams, A. (2013). Information underpinning anticipation of goal-directed throwing. Attention, Perception \& Psychophysics, 75, 1559-1569.

Buckolz, E., Prapavesis, H., \& Fairs, J. (1988). Advance cues and their use in predicting tennis passing shots. Canadian Journal of Sport Sciences = Journal Canadien Des Sciences Du Sport, 13, 20-30.

Cañal-Bruland, R., \& Mann, D. L. (2015). Time to broaden the scope of research on anticipatory behavior: A case for the role of probabilistic information. Frontiers in Psychology, 6. 
1 Causer, J., Smeeton, N. J., \& Williams, A. M. (2017). Expertise differences in anticipatory 2 judgements during a temporally and spatially occluded task. PloS One, 12, e0171330.

3 Cocks, A. J., Jackson, R. C., Bishop, D. T., \& Williams, A. M. (2015). Anxiety, anticipation 4 and contextual information: A test of attentional control theory. Cognition and Emotion, $5 \quad 30,1037-1048$.

6 Crognier, L., \& Féry, Y. (2005). Effect of tactical initiative on predicting passing shots in 7 tennis. Applied Cognitive Psychology, 19, 637-649.

8 Ericsson, K. A., \& Kintsch, W. (1995). Long-term working memory. Psychological $9 \quad$ Review, 102, 211-245.

Farrow, D., \& Abernethy, B. (2015). Expert anticipation and pattern recognition. In J. Baker \& D. Farrow (Eds.), Routledge Handbook of Sport Expertise (pp. 9-21). Oxon, UK; Routledge.

Farrow, D., \& Reid, M. (2012). The contribution of situational probability information to anticipatory skill. Journal of Science and Medicine in Sport, 15, 368-373.

18 Gigerenzer, G., \& Todd, P. M. (1999). Fast and frugal heuristics: The adaptive toolbox. In Simple heuristics that make us smart (pp. 3-34). Oxford: Oxford University Press. 
1 Gredin, V. G., Bishop, D. T., Broadbent, D. P., Tucker, A., \& Williams, A. M. (2018). Experts integrate contextual priors and environmental information to improve anticipation efficiency. Journal of Experimental Psychology: Applied.

Hoffman, R. R., Ward, P., Feltovich, P. J., DiBello, L., Fiore, S. M., \& Andrews, D. H.

5 (2014). Accelerated expertise: Training for high proficiency in a complex world. Hove:

6 Psychology Press.

7 Huys, R., Cañal-Bruland, R., Hagemann, N., Beek, P. J., Smeeton, N. J., \& Williams, A. M. (2009). Global information pickup underpins anticipation of tennis shot direction. Journal of Motor Behavior, 41, 158-170.

Jackson, R. C., \& Mogan, P. (2007). Advance visual information, awareness, and anticipation skill. Journal of Motor Behavior, 39, 341-351.

Jackson, R. C., Warren, S., \& Abernethy, B. (2006). Anticipation skill and susceptibility to deceptive movement. Acta Psychologica, 123, 355-371.

Johnson, J. G., \& Raab, M. (2003). Take the first: Option-generation and resulting choices. Organizational Behavior and Human Decision Processes, 91, 215-229.

Laborde, S., \& Raab, M. (2013). The tale of hearts and reason: The influence of mood on decision making. Journal of Sport and Exercise Psychology, 35, 339-357.

Loffing, F., Sölter, F., Hagemann, N., \& Strauss, B. (2015). Accuracy of outcome anticipation, but not gaze behavior, differs against left-and right-handed penalties in team-handball goalkeeping. Frontiers in Psychology, 6, 1820. 
1 Loffing, F., \& Hagemann, N. (2014). On-court position influences skilled tennis players' anticipation of shot outcome. Journal of Sport \& Exercise Psychology, 36, 14-26.

Mann, D. T. Y., Williams, A. M., Ward, P., \& Janelle, C. M. (2007). Perceptual-cognitive expertise in sport: A meta-analysis. Journal of Sport \& Exercise Psychology, 29, $457-$ 478.

McRobert, A. P., Ward, P., Eccles, D. W., \& Williams, A. M. (2011). The effect of manipulating context-specific information on perceptual-cognitive processes during a simulated anticipation task. British Journal of Psychology (London, England: 1953), 102, 519-534.

McRobert, A., Williams, A. M., Ward, P., \& Eccles, D. (2009). Tracing the process of expertise in a simulated anticipation task. Ergonomics, 52, 474-483.

Mecheri, S., Rioult, F., Mantel, B., Kauffmann, F., \& Benguigui, N. (2016). The serve impact in tennis: First large-scale study of big Hawk-Eye data. Statistical Analysis and Data Mining: The ASA Data Science Journal, 9, 310-325.

Milazzo, N., Farrow, D., Ruffault, A., \& Fournier, J. F. (2015). Do karate fighters use situational probability information to improve decision-making performance during onmat tasks? Journal of Sports Sciences, 1-10.

Müller, S., \& Abernethy, B. (2012). Expert anticipatory skill in striking sports: A review and a model. Research Quarterly for Exercise and Sport, 83, 175-187.

Müller, S., Abernethy, B., Eid, M., McBean, R., \& Rose, M. (2010). Expertise and the spatiotemporal characteristics of anticipatory information pick-up from complex movement patterns. Perception, 39, 745-760. 
1 Murphy, C. P., Jackson, R. C., Cooke, K., Roca, A., Benguigui, N., \& Williams, A. M. (2016). Contextual information and perceptual-cognitive expertise in a dynamic, temporally-constrained task. Journal of Experimental Psychology: Applied, 22, 455-470.

Roca, A., Ford, P. R., McRobert, A. P., \& Williams, A. M. (2011). Identifying the processes

4 Murphy, C. P., Jackson, R. C., \& Williams, A. M. (2018). The role of contextual information 5 6 during skilled anticipation. Quarterly Journal of Experimental Psychology, 71, 20702087.

7 Navia, J. A., van der Kamp, J., \& Ruiz, L. M. (2013). On the use of situational and body information in goalkeeper actions during a soccer penalty kick. International Journal of Sport Psychology, 44, 234-251.

North, J. S., Hope, E., \& Williams, A. M. (2016). The relative importance of different perceptual-cognitive skills during anticipation. Human Movement Science, 49, 170-177.

Raab, M. (2012). Simple heuristics in sports. International Review of Sport and Exercise Psychology, 5, 104-120.

Raab, M., \& Johnson, J. G. (2007). Expertise-based differences in search and optiongeneration strategies. Journal of Experimental Psychology: Applied, 13, 158-170.

Raab, M., \& Laborde, S. (2011). When to blink and when to think: preference for intuitive decisions results in faster and better tactical choices. Research Quarterly for Exercise and Sport, 82, 89-98. task. Cognitive Processing, 12, 301-310. 
1 Roca, A., Ford, P. R., McRobert, A. P., \& Williams, A. M. (2013). Perceptual-cognitive skills and their interaction as a function of task constraints in soccer. Journal of Sport \& Exercise Psychology, 35, 144-155.

4 Savelsbergh, G. J., Williams, A. M., Kamp, J. V. D., \& Ward, P. (2002). Visual search, anticipation and expertise in soccer goalkeepers. Journal of Sports Sciences, 20, 2796287.

7 Sebanz, N., \& Shiffrar, M. (2009). Detecting deception in a bluffing body: The role of expertise. Psychonomic Bulletin \& Review, 16, 170-175.

Triolet, C., Benguigui, N., Le Runigo, C., \& Williams, A. M. (2013). Quantifying the nature of anticipation in professional tennis. Journal of Sports Sciences, 31, 820-830.

Ward, P., Ericsson, K. A., \& Williams, A. M. (2013). Complex perceptual-cognitive expertise in a simulated task environment. Journal of Cognitive Engineering and Decision Making, 7, 231-254.

Ward, P., Suss, J., Eccles, D. W., Williams, A. M., \& Harris, K. R. (2011). Skill-based differences in option generation in a complex task: A verbal protocol analysis. Cognitive Processing, 12, 289-300.

7 Ward, P., Williams, A. M., \& Bennett, S. J. (2002). Visual search and biological motion perception in tennis. Research Quarterly for Exercise and Sport, 73, 107-112.

Williams, A. M., \& Burwitz, L. (1993). Advance cue utilization in soccer. In T. Reilly, J. Clarys, \& A. Stibbe (Eds.), Science and Football II (pp. 239-244). London: E \& FN Spon. 
1 Williams, A. M., Davids, K., \& Williams, J. G. P. (1999). Visual perception and action in sport. New York: E \& FN Spon.

8 
1 Tables

2 Table 1. A summary of the main statistical test results.

\begin{tabular}{lrrrr}
\hline Effect & $d f$ & $F$ & $p$ & $\eta_{p}^{2}$ \\
\cline { 1 - 1 } Anticipation Accuracy & & & & \\
\cline { 1 - 4 } Display & 1,24 & 21.08 & $<.01$ & 0.47 \\
Group & 1,24 & 24.21 & $<.01$ & 0.50 \\
Display $\times$ Group & 1,24 & 18.87 & $<.01$ & 0.44 \\
\multicolumn{1}{l}{ Option Generation: Number and Type } & & & & \\
Display & 1,24 & 23.90 & $<.01$ & 0.50 \\
Group & 1,24 & 0.78 & 0.39 & 0.03 \\
Option Type & 1,24 & 0.41 & 0.53 & 0.02 \\
Group $\times$ Option Type & 1,24 & 37.25 & $<.01$ & 0.61 \\
Display $\times$ Option Type & 1,24 & 15.37 & $<.01$ & 0.39 \\
Display $\times$ Group & 1,24 & 3.67 & 0.07 & 0.13 \\
Display $\times$ Group $\times$ Option Type & 1,24 & 5.08 & 0.03 & 0.18 \\
\hline
\end{tabular}

3

4

5

6

7

8

9

10 
1 Table 2. The correlations between number of generated options and anticipation accuracy in

2 video and animated display conditions.

\begin{tabular}{lccccccccccc}
\hline & \multicolumn{1}{c}{ Number of Generated Options } \\
\cline { 2 - 13 } & \multicolumn{1}{c}{ All } & \multicolumn{1}{c}{ Expert } & Novice & \multicolumn{2}{c}{$\begin{array}{c}\text { Task- } \\
\text { Relevant }\end{array}$} & \multicolumn{2}{c}{$\begin{array}{c}\text { Task- } \\
\text { Irrelevant }\end{array}$} \\
\cline { 2 - 14 } Condition & $r$ & $p$ & $r$ & $p$ & $R$ & $p$ & $r$ & $p$ & $r$ & $p$ \\
\hline Video & -0.44 & 0.01 & -0.22 & 0.25 & -0.46 & 0.05 & 0.35 & 0.04 & -0.62 & $<.01$ \\
Animated & -0.25 & 0.11 & -0.17 & 0.30 & -0.34 & 0.12 & 0.38 & 0.03 & -0.60 & $<.01$ \\
\hline
\end{tabular}

3

4

5

6

7

8

9

10 


\section{Figure Captions}

2 Figure 1. An example of the video (top) and animated (bottom) display conditions employed.

4 Figure 2. The mean (SE) anticipation accuracy of expert and novice participants in video and 5 animated display conditions.

7 Figure 3. The mean (SE) number of task-relevant and task-irrelevant options generated per

8 trial by expert and novice participants in video and animated display conditions.

10 Figure 4. The relationship between mean number of options generated and accuracy of expert 11 and novice participants in video (a) and animated (b) display conditions.

13 Figure 5. The relationship between number of task-relevant and task-irrelevant options

14 generated and accuracy in video (a) and animated (b) display conditions. 


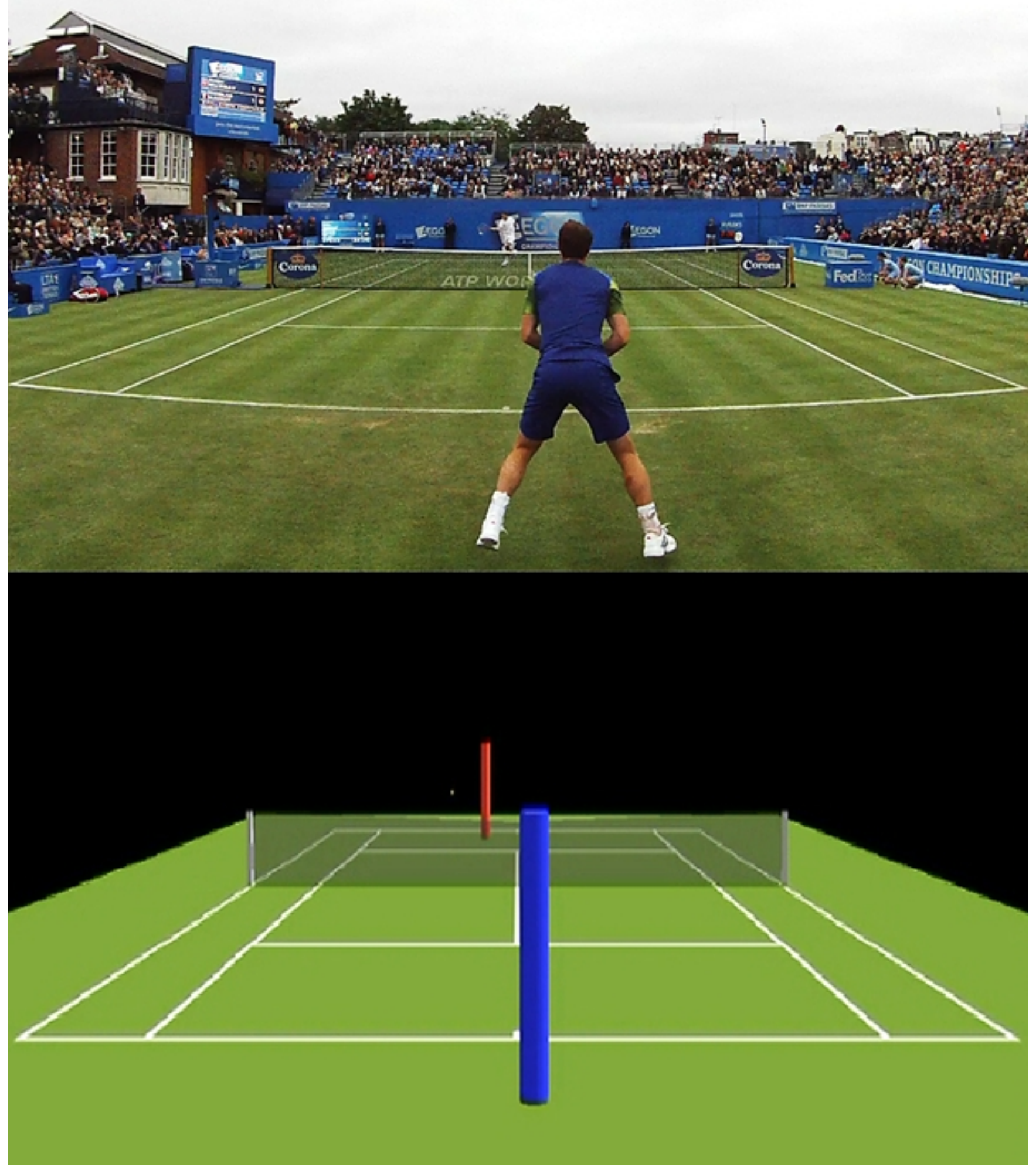




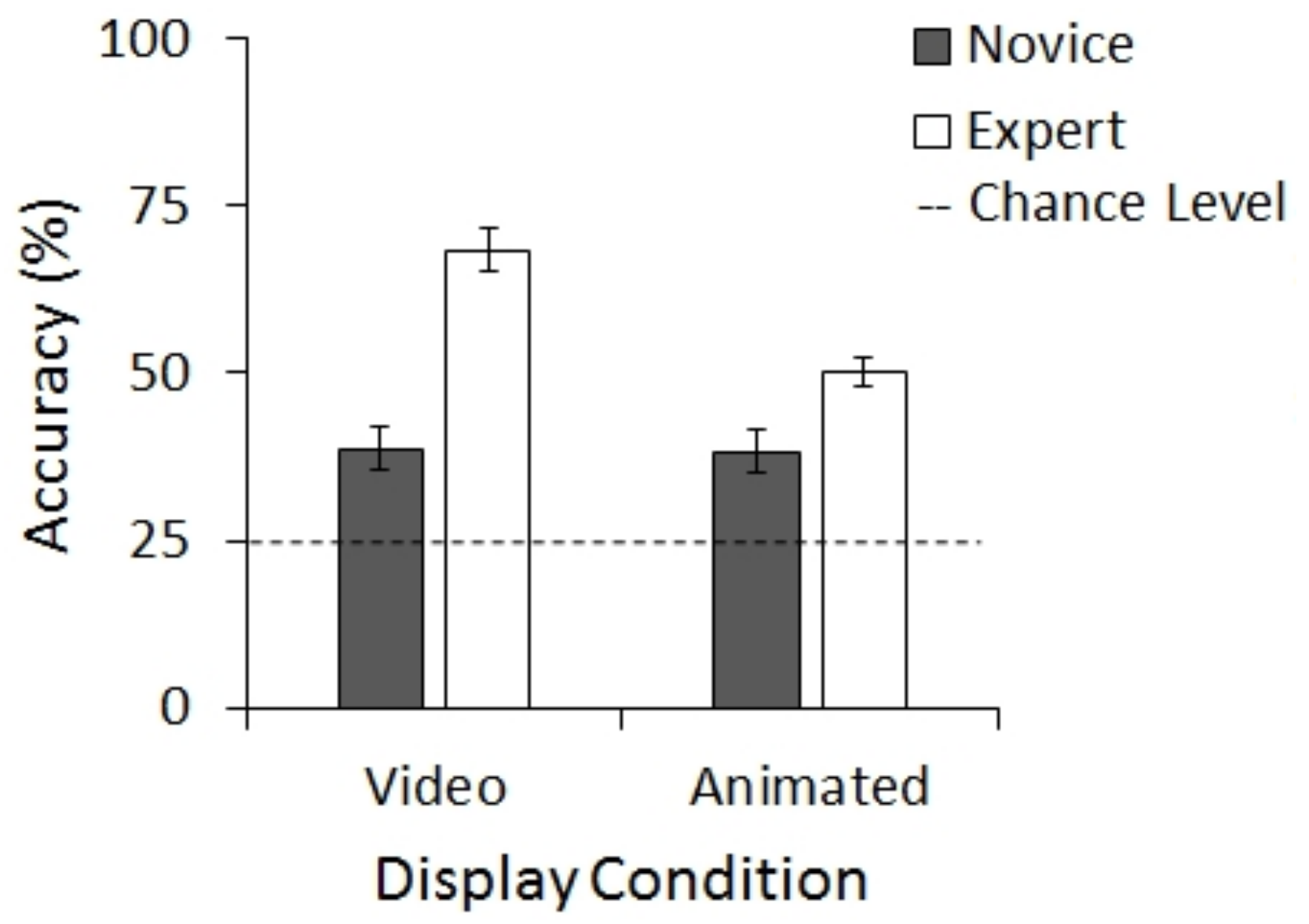




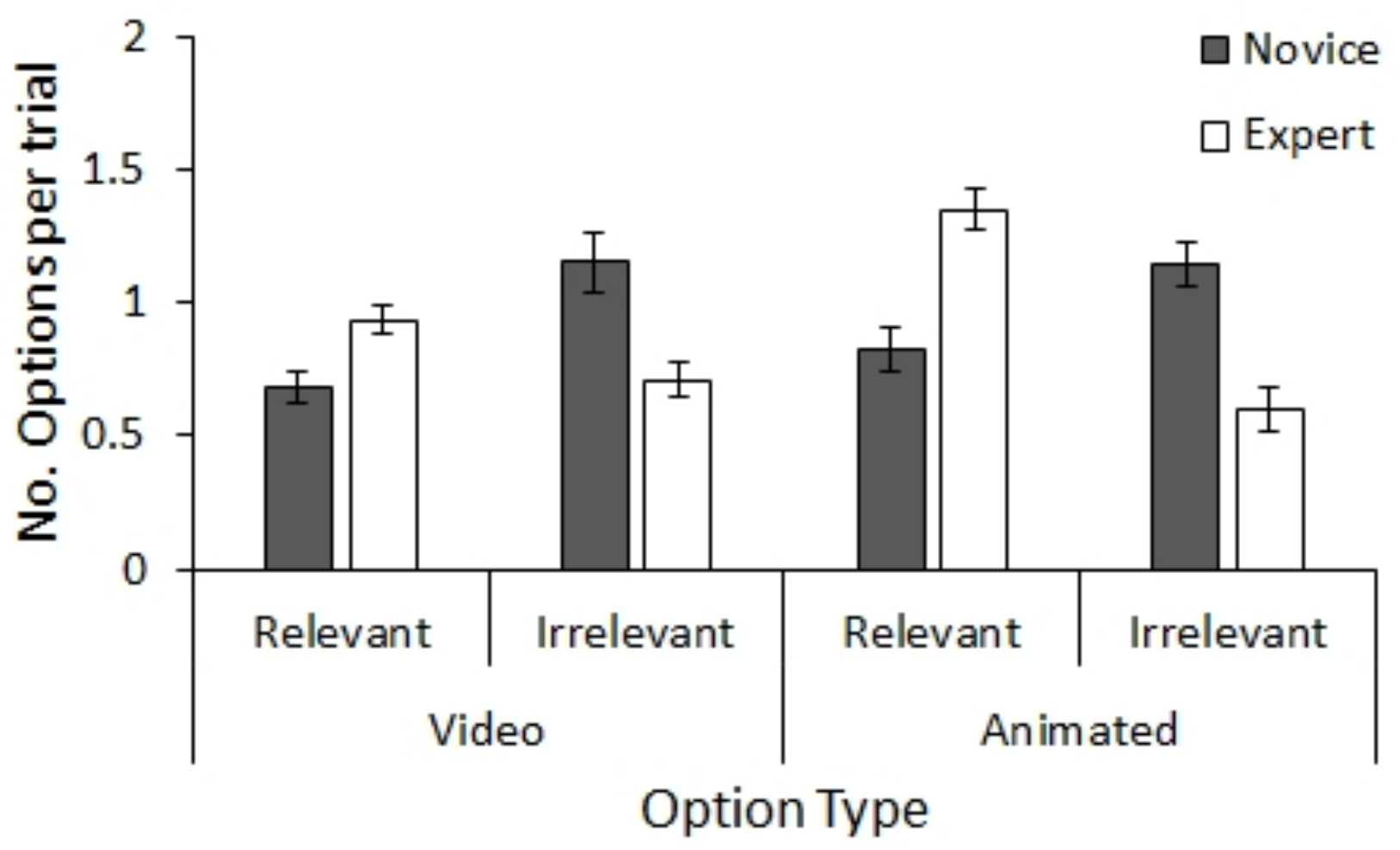




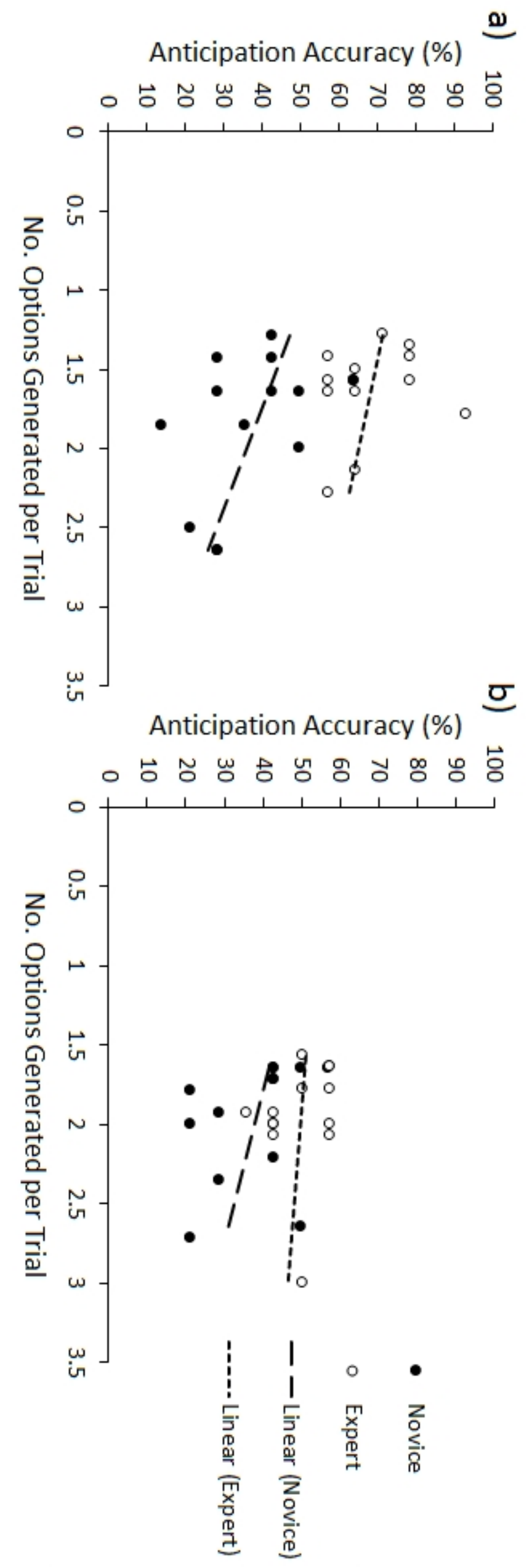




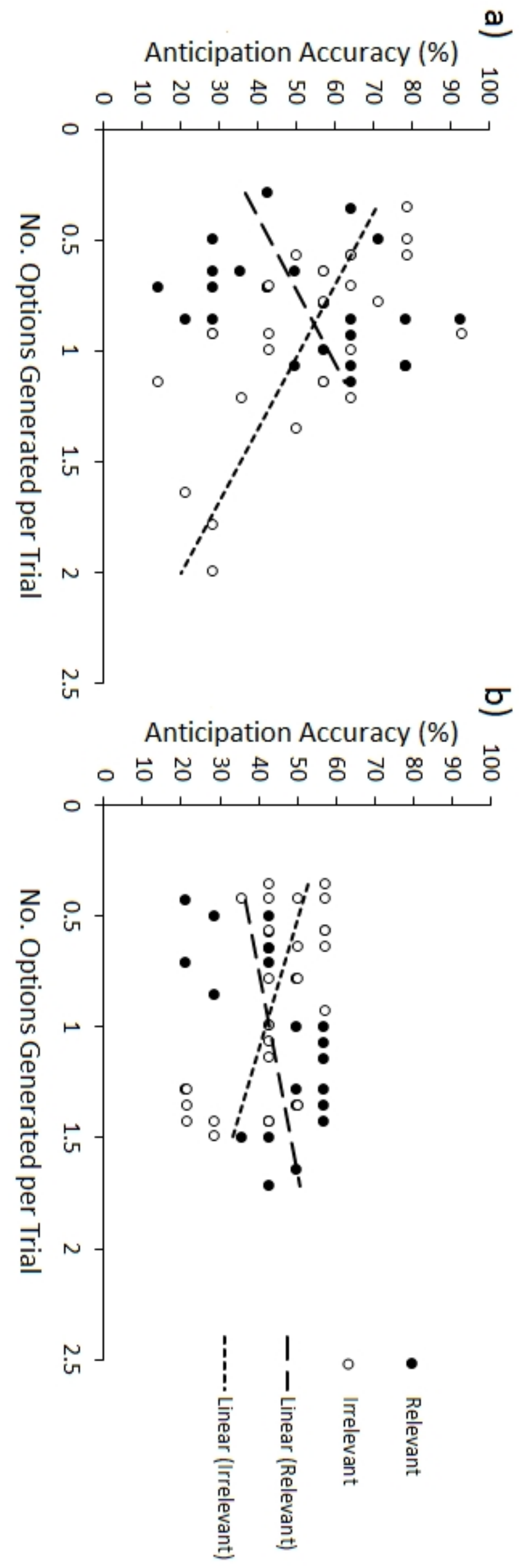




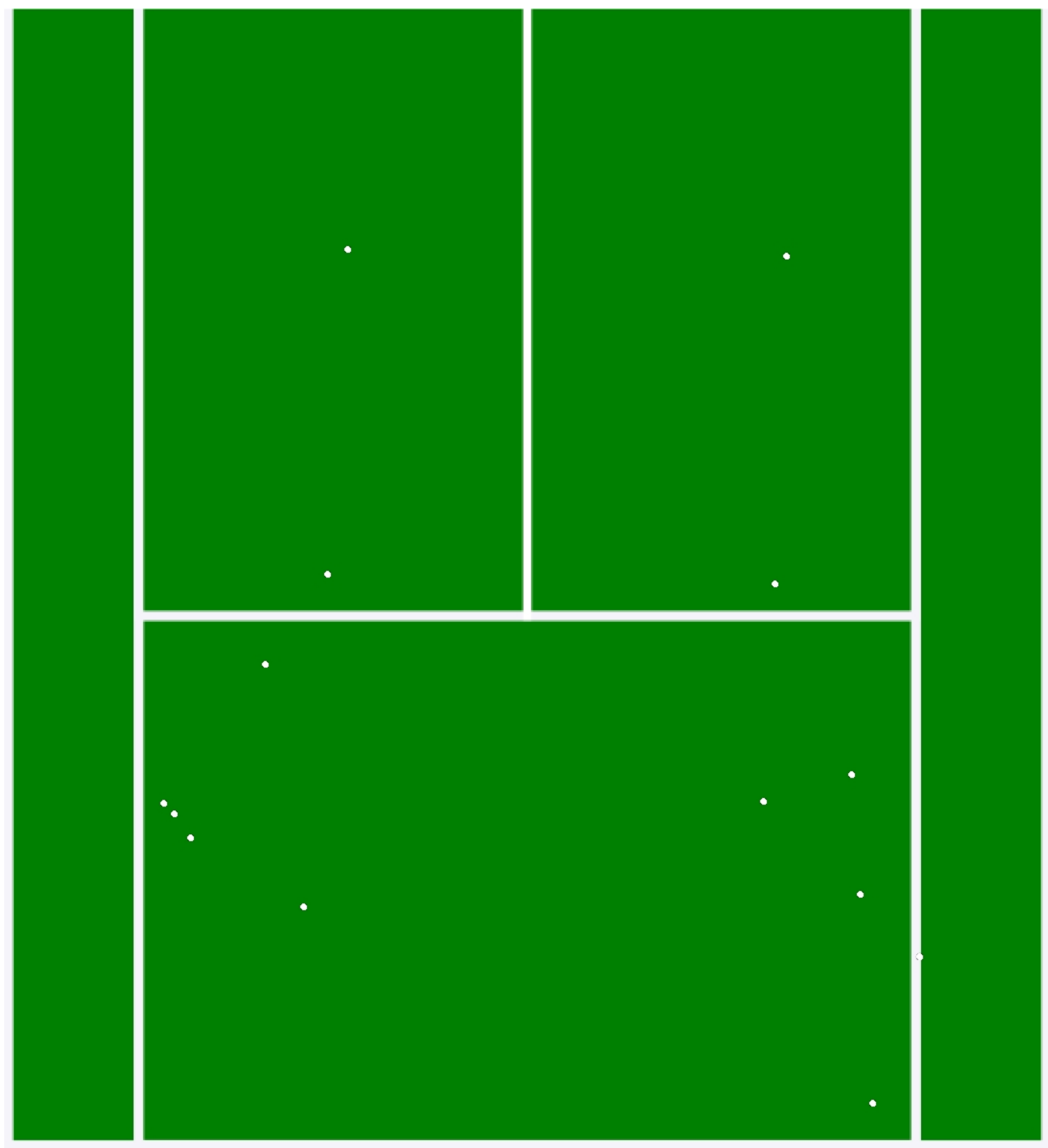

